\title{
BEGLEITWORT ZUR DEUTSCHEN ÜBERSETZUNG
}

Von bedeutenden, umfassenden Darstellungen der Chemie des Holzes existieren augenblicklich in der Weltliteratur lediglich drei:

E. HägGlund, Chemistry of Wood; L. E. Wise, Wood Chemistry und

Н. И. Нинитин, Химия древесины.

Nachdem das Buch von N. I. Nikitin im Jahre 1951 neubearbeitet erschienen ist, hielt ich es für wünschenswert, eine Übersetzung aus dem Russischen in das Deutsche dieser Darstellung der Chemie des Holzes durchführen zu lassen. Dafür waren unter anderen folgende Gründe maßgebend :

E. HÄGGLUND hat seine Holzchemie, die 1928 zuerst und dann 1939 in zweiter, unveränderter Auflage erschienen war, nicht mehr in deutscher, sondern 1951 in Amerika in englischer Sprache vollkommen neu bearbeitet herausgebracht; es fehlt deshalb eine größere zusammenfassende neuere Darstellung dieses Gebietes in deutscher Sprache.

In den letzten 35 Jahren ist in der Sowjetunion der Bearbeitung der Chemie des Holzes besondere Beachtung geschenkt worden. Dies zeigt sich in einer groBen Anzahl von Veröffentlichungen sowjetischer Wissenschaftler, die neben ihrer wissenschaftlichen Bedeutung für die Entwicklung der sehr wichtigen Verwendung und Verwertung des Holzes große Bedeutung haben. Da in den Büchern von E. HÄGGLUND und L.E. WISE diese sowjetischen Arbeiten überhaupt nicht oder nur in ganz ungenügendem Maße (8 Literaturstellen bei HäGGLUND) berücksichtigt worden sind, dürfte eine deutsche Übersetzung des Nikitinschen Werkes, das gerade auch die neueren sowjetischen Arbeiten auf dem Gebiete der Holzchemie berücksichtigt, für uns von besonderer Bedeutung sein und auch für die gesamte nicht russisch sprechende oder verstehende wissenschaftliche Welt, die die Resultate dieser Arbeiten nur aus meist mehr oder weniger verständlichen Kurzreferaten kennt, Interesse haben.

Die Ưbertragung wurde von einem Kollektiv aus Mitgliedern der Abteilung Cellulose-Zellstoff des Institutes für Faserstoff-Forschung der Deutschen Akademie der Wissenschaften zu Berlin in Teltow-Seehof ausgeführt. Es bestand aus Herrn Wittwer, dem fachlich erfahrenen Übersetzer, und den Herren Dr. Edelmann und Dr. Schorning, die eine redaktionelle und wissenschaftliche Überprüfung der Übersetzung übernahmen.

\section{ERICH CORRENS}

Mitglied der Deutschen Akademie der

Wissenschaften und Direktor des Institutes für Faserstoff-Forschung. 
